\title{
Kooperativer Wettbewerb im Zeitalter der DRGs
}

\section{Clarissa Kurscheid ${ }^{a}$ \\ Reinhard Voegele ${ }^{b}$}

a Prof. Dr., Dekanin für Gesundheitsökonomie an der Hochschule Fresenius in Köln

b lic. rer. pol., H+ Die Spitäler der Schweiz
Korrespondenz:

lic. rer. pol. Reinhard Voegele Leiter Kommunikation

H+ Die Spitäler der Schweiz Geschäftsstelle

Lorrainestrasse $4 \mathrm{~A}$

CH-3013 Bern

reinhard.voegele@hplus.ch
Die Schweizer Spitäler ändern im kommenden Jahr ihr Vergütungssystem. Mit dieser Entscheidung reagiert die Schweiz ähnlich wie andere Staaten auf die Folgen des demografischen Wandels und die steigenden Krankheitskosten - nicht zuletzt bedingt durch den medizinisch technischen Fortschritt - und eine sich anbahnende Ressourcenknappheit. Die DRGs (Diagnosis Related Groups - diagnosebezogene Fallpauschalen) sind ein verbindlicher politischer Auftrag an das Gesundheitssystem. Somit sind die Spitäler derzeit in hohem Masse damit beschäftigt, diesen Auftrag zu erfüllen. DRGs anzuwenden und sie zu verwirklichen; das findet auf der konkreten, operationellen Ebene statt. Damit lautet das Ziel für die Spitäler: Die vorhandenen Strukturen sind so zu verändern, dass sie trotz veränderter Vergütung eine adäquate Versorgung sicherstellen.

Die Schritte zur Verwirklichung der neuen Strukturen haben die Verantwortlichen in der Schweiz in den letzten Jahren konsequent realisiert, sie haben die Leistungsdichte erhöht und sich mit tradierten Prozessen auseinandergesetzt. Es fanden hauseigene Untersuchungen statt. Mit dem neuen System kommen jedoch zusätzliche Fragen auf:

- Kann ein Vergütungssystem massgeblichen Einfluss auf medizinisches Handeln nehmen?

- Sind schlechtere Behandlungsqualität und mit Unterbrüchen behaftete Behandlungsprozesse eine Folge des wirtschaftlichen Handelns?

In der Vorausschau auf die Arbeit mit Fallpauschalen werden insbesondere die Gefahr der Verringerung der medizinischen Qualität und mögliche Drehtür- oder Weiterreichungseffekte benannt. Die Kritiker argumentieren in diesem Zusammenhang mit einem höheren Leistungsvolumen und mit geringeren zur Verfügung stehenden Zeiträumen für die tätigen Akteure.

\section{Nach wie vor: Menschen wieder fit machen für den Alltag}

Folglich steht ein Paradigmenwechsel an, der eine andere Herangehensweise fordert. Ein veränderter Umgang mit Behandlungsprozessen und veränderte Zeitressourcen beinhalten nicht automatisch verschlechterte Arbeitssituationen für das Personal, dies sei jedoch nur am Rande erwähnt. Die Kernaufgabe, eine Person gesund oder wieder so weit wie möglich fit zu machen, dass sie ihren Alltag meistern kann, bleibt bestehen. Sie muss jedoch nicht zwingend aus einer Hand und auch nicht innerhalb einer einzigen Fachabteilung eines Spitals geschehen. Die Branche Gesundheitswesen entwickelt nun neben dem Moment der Spezialisierung einen stärkeren Blick auf die Arbeitsteilung. Ihr soll im Folgenden Aufmerksamkeit geschenkt werden.
- Wie viel soll man in welchem Abschnitt des Versorgungsprozesses leisten, und welche Tätigkeiten erfolgen in vor- oder nachgelagerten Prozessabschnitten?

Bevor für diese Frage unter Beachtung des kooperativen Wettbewerbs Antworten gefunden werden, erfolgt ein Blick auf die Situation unter der neuen Vergütungssystematik. Unter DRG-Regime bedeutet ein Arbeiten mit Fallpauschalen eine Spezialisierung auf die Kernkompetenzen und Kostentransparenz. Vergütet werden die Mengen der codierten Prozeduren und Prozesse, die unter die jeweilige DRG fallen. Was bedeutet das für die Spitäler? Bei potentiellem Wunsch nach Ertragsmaximierung sollte ein Spital orientiert am Case-Mix mit den entsprechenden Häufigkeiten eine Spezialisierung verfolgen. Dies kann ein zukünftiges strategisches Ziel sein. Wir erwarten bei einer Spezialisierung von Kernkompetenzen eine steigende Häufigkeit von bestimmten Eingriffen. Die daraus resultierende, zusätzliche Erfahrung mündet in einer steigenden Behandlungs- und Versorgungsqualität.

Unter Beachtung bestehender Qualitätssicherungsmassnahmen ist davon auszugehen, dass die effektive Nutzung von Kernkompetenzen die Behandlungsqualität erhöht und Fehler minimiert.

Die Stärkung und die Nutzung von Kernkompetenzen ist somit ein Ergebnis aus der Veränderung der Vergütungssystematik. Allein: Was geschieht, wenn ich nur noch Spezialitäten behandle, ist dann eine breitflächige Versorgung nicht mehr machbar?

Hier ist für die nahe und mittelfristige Zukunft ein Umdenken der Spitalleitung gefordert. Da die Spitalleitungen nicht ausschliesslich teure Spezialisierungen einkaufen können und dürfen, sind sie gefordert, mit anderen spezialisierten Clustern zu kooperieren, um gleichzeitig ihren Auftrag einer flächendeckenden Versorgung zu gewährleisten. Somit geht es in der Zukunft stark um die

\section{Rolle der Spitäler in Integrierten Versorgungsnetzen}

Ziel der Versorgungsnetze ist es, den Patientinnen und Patienten qualitativ bessere Behandlungen zuteil werden zu lassen und dies über Kooperationen zu gewährleisten. Demnach muss es ein Ziel der Spitäler und der Spitalleitungen sein, dass die verschiedenen Leistungserbringer im Gesundheitswesen besser zusammenarbeiten.

Folglich entstehen hier wiederum zwei Thesen, die es zu betrachten gilt:

- Spezialisierte Cluster erfordern Kooperationen zum gelingenden Handeln und zur flächendeckenden Versorgung. 
- Gute Kooperationen überwinden potentielle Verzögerungs- und Vermeidungseffekte zwischen Spezialisierung und Regionalisierung, denn es entsteht ein ganz neues Risiko der mangelnden Basisversorgung ohne Unterbrüche.

\section{Behandlungsqualität erfordert eine neue Qualität der Kooperation}

Für die einzelnen Spitäler bedeutet dies, mit einer effizienten und somit am Ertrag ausgerichteten Vergütung Versorgungsprozesse transparent und möglichst ohne Unterbrüche zu verwirklichen. Die zu entwickelnden Versorgungsprozesse sind zudem interprofessionell und intersektoral zu gestalten. Wesentliche Merkmale sind neben einer veränderten Struktur insbesondere tragfähige Kommunikationsprozesse. Nur dann ist gewährleistet, dass Patienten innerhalb einer festgelegten Verweildauer so behandelt und therapiert werden, dass sie wieder am Alltag teilnehmen können. Das gesellschaftliche Leben erfordert aber aufgrund seiner Komplexitäten nicht zuletzt im Erkrankungsfall mehr als nur medizinische Versorgung. Hier taucht eine erhöhte Schnittstellenproblematik insbesondere ausserhalb des Spitalbereichs auf. Möchten die Spitäler zusätzlich Behandlungsqualität bieten und Drehtür- oder Weiterreichungseffekte verhindern, wird das Thema Kooperation für sie unvermeidlich.

Literaturhinweise finden sich im Internet unter www.saez.ch $\rightarrow$ Aktuelle Nummer oder $\rightarrow$ Archiv $\rightarrow 2011 \rightarrow 36$

\section{Kooperation und Konkurrenz}

Die Spitäler stehen im Wettbewerb um den Patienten. Sie konkurrenzieren sich. Der Wettbewerb findet unter den Spitälern indirekt über Budgets statt. In Zu- kunft werden die Verhandlungen bei den jährlichen Budgets verstärkt auf Mengenfragestellungen ausgerichtet sein. Um sich dabei attraktiv aufzustellen, müssen die Spitalleitungen den ökonomischen Mehrwert von Offenheit und Kooperationen erst einmal erkennen.

\section{Fazit}

Zusammenfassend können wir festhalten, dass Spitäler unter den zukünftigen Bedingungen kaum andere Möglichkeiten haben, als Kooperationen einzugehen. Mit Kooperationen überwinden die Spitäler fragmentierte Behandlungsprozesse und generieren eine grössere Behandlungsqualität bei Kosteneinsparungen. Neben einer Arbeitsteilung steht zukünftig die Koordination der kooperierenden Unternehmen orientiert am sektorenübergreifenden Behandlungsprozess im Vordergrund. Es braucht ein wettbewerbsorientiertes Denken der beteiligten Akteure als treibende Kraft für die Entstehung von Kooperationen.

\section{H+}

Besuchen Sie den H+ Kongress 2011

zum Thema «Integrierte Behandlungsnetze: Kooperation und Konkurrenz"

3. November 2011, Hotel Bellevue Palace, Bern

Weitere Informationen und die Anmeldung finden Sie unter www.hplus-kongress.ch
Diskutieren Sie mit: Schreiben Sie Ihre Meinung mit einem Kommentar im öffentlichen Diskussionsforum auf der Webseite www.hplus-kongress.ch unter der Rubrik «Diskutieren Sie mit».

\begin{abstract}
Drei theoretische Ansätze zu Kooperationen
Kooperationen können in der Theorie nicht über einen ganzheitlichen Ansatz erläutert werden, dennoch bieten sich einige Erklärungsansätze an, die sich auf die Entstehung von Kooperationen, die Struktur und Organisation der Zusammenarbeit und auf die Begründung kooperativer Wettbewerbsvorteile beziehen. Nachfolgend werden anhand von Kooperationszielen die bekanntesten Erklärungsansätze, der markt- und ressourcenorientierte sowie der spieltheoretische Ansatz, kurz erläutert.
\end{abstract}

\section{Der marktorientierte Ansatz}

Der marktorientierte Ansatz, der aus der Institutionenökonomie herrührt, verfolgt den Gedanken, dass Unternehmenskonzentrationen oder Eintrittsbarrieren bestimmte Wirkungen auf das strategische Verhalten von Unternehmen haben. Die daraus resultierenden Kooperationsziele sind die Schaffung von Wettbewerbsvorteilen, die Optimierung der Wettbewerbsposition und die Veränderung von Marktstrukturen. Der Ansatz erklärt unter besonderer Beachtung der Verhaltenskomponente der beteiligten Akteure die Entstehung von sektorübergreifenden Kooperationen als Reaktion auf dynamische Entwicklungen der Rahmenbedingungen. Damit leistet der Erklärungsansatz einen wesentlichen Beitrag, neue ökonomische Dimensionen von Kooperationsumfeldern darlegen zu können. Er gibt jedoch keine Informationen zu den organisationalen Strukturen von Kooperationen und ist für die interne Unternehmensplanung nur begrenzt hilfreich.

\section{Der ressourcenorientierte Ansatz}

Der ressourcenorientierte Ansatz geht im Gegensatz zum vorher erläuterten Konzept nicht über die marktlichen Entwicklungsgedanken vor, sondern behauptet, dass ein langfristiger Erfolg von Unternehmen nicht über ein Agieren auf dem Markt, sondern über die effektive Ausnutzung der eigenen Ressourcenausstattung und die Qualität dessen zu erklären ist. In der Betrachtung der Ressourcen werden materielle wie auch immaterielle einbezogen. Für das Gesundheitswesen kann die strategische Betrachtung mit Hilfe dieses Ansatzes von hoher Relevanz sein, da das Gesundheitswesen zu den wissensintensiven Dienstleistungen zählt und die immaterielle Ressource Wissen ein wesentliches Merkmal im Wettbewerb ist.

\section{Der spieltheoretische Ansatz}

Der spieltheoretische Ansatz hingegen betrachtet die internen Handhabungen von Kooperationen und die sozialen Beziehungen innerhalb einer Kooperation. Maximierungen des Kooperationsertrags und die Erhöhung der Kooperationsstabilität lassen sich insbesondere in horizontal geprägten Kooperationen, beispielsweise zwischen zwei Spitälern, gut erklären. Alle genannten Ansätze bieten sowohl aus Marktsicht als auch aus Unternehmenssicht Erklärungsmöglichkeiten für kooperative Beziehungen und deren Auswirkungen. Die theoretischen Ansätze sind allerdings nicht alleine für sich in der Lage, ein umfassendes Verständnis über eine Kooperation im Gesundheitswesen zu entwickeln. In der Gesamtheit bieten sie aber für das Verständnis erweiternde Muster an. 


\section{Literaturhinweise}

Barney JB. Strategic Factor Markets: Expectations, Luck and Business Strategy. Management Science. 1986;32:1231-41.

Barney JB. Firm Resources and Sustained Competitive Advantage. Journal of Management. 1991;17:99-120.

Friese M. Kooperation als Wettbewerbstrategie für Dienstleistungsunternehmen. Wiesbaden; 1998.

Porter ME. Wettbewerbsstrategie: Methoden zur Analyse von Branchen und Konkurrenten. Frankfurt a.M.;1999.

Porter M E Competitive Strategy: Techniques for Analyzing Industries and Competitors. New York; 1980.

Schlüchtermann J, Albrecht M. Private Konzerne als Schrittmacher für das Krankenhaus im Wandel. In: Hellmann W. Handbuch Integrationsversorgung. 2006; S. 1-23.

Wohlgemut O. Management netzwerkartiger Kooperationen - Instrumente für die unternehmensübergreifende Steuerung. Wiesbaden; 2002). 